OPEN ACCESS

Edited by:

Mor-Li Hartman,

The Forsyth Institute, United States

Reviewed by:

Sepideh Babaniamansour, University of Michigan, United States Ishag Adam,

University of Khartoum, Sudan

${ }^{*}$ Correspondence:

Lixin Yang

yanglx282@sina.com

Xiaoxing Wei

weixiaoxing@tsinghua.org.cn

${ }^{t}$ These authors have contributed equally to this work and share first authorship

Specialty section: This article was submitted to

Clinical Diabetes, a section of the journal

Frontiers in Endocrinology

Received: 03 July 2021 Accepted: 24 December 2021 Published: 04 February 2022

Citation:

Liu Y, Gong R, Luo G, Li J, Li Q, Yang L and Wei $X$ (2022) Associations of Triglycerides/High-Density Lipoprotein Cholesterol Ratio With Insulin

Resistance, Impaired Glucose Tolerance, and Diabetes in American Adults at Different Vitamin D3 Levels.

Front. Endocrinol. 12:735736. doi: 10.3389/fendo.2021.735736

\section{Associations of Triglycerides/ High-Density Lipoprotein Cholesterol Ratio With Insulin Resistance, Impaired Glucose Tolerance, and Diabetes in American Adults at Different Vitamin D3 Levels}

\author{
Yuanyuan Liu ${ }^{1,2 \dagger}$, Rongpeng Gong ${ }^{1 \dagger}$, Gang $\mathrm{Luo}^{3 \dagger}$, Jinxia $\mathrm{Li}^{1,2}$, Qidan $\mathrm{Li}^{1}$, \\ Lixin Yang ${ }^{1,2^{*}}$ and Xiaoxing Wei ${ }^{1,3^{*}}$ \\ ${ }^{1}$ Medical College of Qinghai University, Xining, China, ${ }^{2}$ Endocrinology Department, Qinghai Provincial People's Hospital,
Xining, China, ${ }^{3}$ College of Eco-environmental Engineering, Qinghai University, Xining, China
}

Background: Previous studies have shown that vitamin D3 (VD3) may be a protective factor for diabetes mellitus (DM), while triglycerides/high-density lipoprotein (TG/HDL) may be a risk factor for diabetes. However, no existing study has elucidated the interaction between TG/HDL and VD3. Therefore, this work aimed to investigate the relationships of $\mathrm{TG} / \mathrm{HDL}$ with insulin resistance (IR), impaired glucose tolerance (IGT), and DM at different VD3 levels.

Methods: With the use of the data from five National Health and Nutrition Examination Survey (NHANES) cycles, a total of 2,929 males and 3,031 females were divided into 4 groups according to their VD3 levels. Logistic regression was performed to observe the associations of TG/HDL ratio with IR, IGT, and DM in different groups.

Results: The relationships of TG/HDL with IR, IGT, and DM showed a threshold effect, with the cutoff values of 1.094, 1.51, and 1.11, respectively. On both sides of the cutoff values, the correlation was first weakened and then enhanced with the increase in VD3 levels.

Conclusion: $T G / H D L$ is a risk factor for $I R, I G T$, and $D M$. Both too low and too high levels of VD3 can strengthen this association, whereas keeping VD3 at a reasonable level helps to reduce the associations of TG/HDL with IR, IGT, and DM.

Keywords: TG/HDL ratio, diabetes mellitus, insulin resistance, impaired glucose tolerance (IGT), crosssectional study

Abbreviations: IR, insulin resistance; IGT, impaired glucose tolerance; DM, diabetes mellitus; EDU, education; SMOK, smoking; BMI, body mass index; ALT, alanine aminotransferase; ALB, albumin; AST, aspartate aminotransferase; ALP, alkaline phosphatase; BUN, blood urea nitrogen; GGT, $\gamma$-glutamyl transpeptidase; UA, uric acid; FPG, fasting plasma glucose; TC, total cholesterol. 


\section{BACKGROUND}

Vitamin D3 (VD3), also called cholecalciferol, is a type of vitamin $\mathrm{D}$ (1). VD3 is the precursor of hormones, which have been recently found to participate in numerous regulatory responses in the body (2-4). Studies have shown that VD3 plays a role not only in bone metabolism but also in insulin resistance (IR), impaired glucose tolerance (IGT), and diabetes mellitus (DM) (3-6).

IR is identified as an impaired response to insulin of target tissues and the resulting reduced efficiency of glucose uptake and utilization (7). If left uncontrolled, IR will develop into IGT and even DM. After feeding rats with a high-fat diet (HFD) for 12 weeks, Zhang et al. found that IR appeared in $93.3 \%$ of the rats (8). With the dramatic changes in people's living conditions, the morbidity of DM shows an increasing trend in various countries worldwide (9-11). According to the Diabetes Atlas 2019 published by the International Diabetes Federation (IDF), there are 425 million patients with type $2 \mathrm{DM}$ (T2DM) around the world (12). According to the current development trend, it is estimated that there will be 629 million DM patients aged 20-79 by the year 2045 , accounting for $10 \%$ of the overall population (13). Besides, there will be more patients with IGT and IR.

At present, VD3 and triglycerides/high-density lipoprotein (TG/HDL) are identified as the factors related to IR, IGT, and DM (14-17). However, few existing studies have investigated whether the relationship between TG/HDL ratio and abnormal glucose metabolism is affected by different VD3 levels. Herein, a retrospective analysis was conducted based on the American National Health and Nutrition Examination Survey (NHANES) database, aiming to discover the difference (or evidence) in the associations of TG/HDL ratio with IR, IGT, and DM at different VD3 levels. The findings in this study will make a significant contribution to exploring the clinical prevention and treatment of DM.

\section{METHODS}

\subsection{Research Population and Test Methods}

Altogether, 49,696 participants from five periods were selected from the NHANES database from 2009 to 2018 . The NHANES project is a subject study strictly formulated by the National Center for Health Statistics (NCHS) to meet the different population representations. NHANES is a persistent project that makes 2 years as a period, and it ensures that the sample is representative of the American population through multilevel and complex sampling design. There are about 5,000 people who receive the sampling survey, which covers diverse aspects like population, social, economy, diet, and health. The laboratory examination section includes medical and physiological tests. All data are collected by professional and trained personnel. NHANES follows a strict standard and protocol to ensure the privacy of each participant, and the information is not used for identification under the U.S. federal law as well.
In this study, strict inclusion and exclusion criteria were applied to select eligible participants. To be specific, participants meeting one or more of the following criteria were excluded: 1) those aged under 18 years; 2) those with no key indicators of insulin, fasting glucose, TG, or HDL; and 3) others (including patients taking drugs that affected blood lipid metabolism, glucose metabolism, and parathyroid metabolism; patients suffering from immunodeficiency, infectious diseases, or malignant tumors; and patients with a recent history of surgery, trauma, severe infection or other stress).

NHANES 2009-2018 covered five periods (2009-2010, 20112012, 2013-2014, 2015-2016, and 2017-2018), involving 49,696 participants in total. Among them, 19,342 participants were excluded due to the age of $<18$ years, 17,313 because of the unavailability of insulin data, 3,374 due to unavailable TG data, and 3,707 because of the lack of HDL data. Finally, 5,960 participants were included in this trial (Figure 1).

Ultrahigh-performance liquid chromatography-tandem mass spectrometry (UHPLC-ms/MS) was performed to quantify VD3 levels in human serum samples. After fasting for $9 \mathrm{~h}$, the fasting plasma glucose (FPG) and insulin levels in the enrolled participants were measured via venipuncture in the morning. Blood lipid data were provided by the laboratory at the University of Minnesota.

In this study, TG/HDL ratio was used as the independent variable, whereas IR, IGT, and DM were used as the dependent variables. According to the definition of VD3, participants were divided into four groups, including VD3 deficiency group (N1, $\mathrm{VD} 3<30 \mathrm{ng} / \mathrm{ml}), \mathrm{VD} 3$ insufficient group $(\mathrm{N} 2,30 \mathrm{ng} / \mathrm{ml}<\mathrm{VD} 3 \leq$ $50 \mathrm{ng} / \mathrm{ml}$ ), VD3 moderate group (N3, $50 \mathrm{ng} / \mathrm{ml}<\mathrm{VD} 3 \leq 80 \mathrm{ng} /$ $\mathrm{ml}$ ), and overdose group (N4, VD3 $\geq 80 \mathrm{ng} / \mathrm{ml}$ ) (Figure 2).

\subsection{Disease Determination}

In this study, the disease determination criterion was strictly formulated based on the international standard.

\subsubsection{Insulin Resistance}

The clinical definition of IR remains elusive, as no generally accepted test is available for IR (18). In some studies, IR is defined by the steady-state model evaluation formula: fasting glucose insulin $(\mu \mathrm{U} / \mathrm{ml}) \times$ fasting glucose $(\mathrm{mmol} / \mathrm{L}) / 22.5(19)$. In other research, IR $>2.6$ is regarded as IR in a normal American population, which was taken as the determination criterion in our study (Figure 2).

\subsubsection{Impaired Glucose Tolerance}

In this study, according to relevant questionnaires and laboratory tests, IGT was defined as self-reported DM or FPG $\geq 6.0 \mathrm{mmol} / \mathrm{L}$ (type $1 \mathrm{DM}$, gestational DM, and specific types of DM were excluded) (20) (Figure 2).

\subsubsection{Diabetes Mellitus}

DM is defined based on the American Diabetes Association Standards 2015. In this study, according to relevant questionnaires and laboratory tests, DM was defined as selfreported DM or glycosylated hemoglobin $\geq 6.5 \%$ and FPG $\geq 6.0$ 


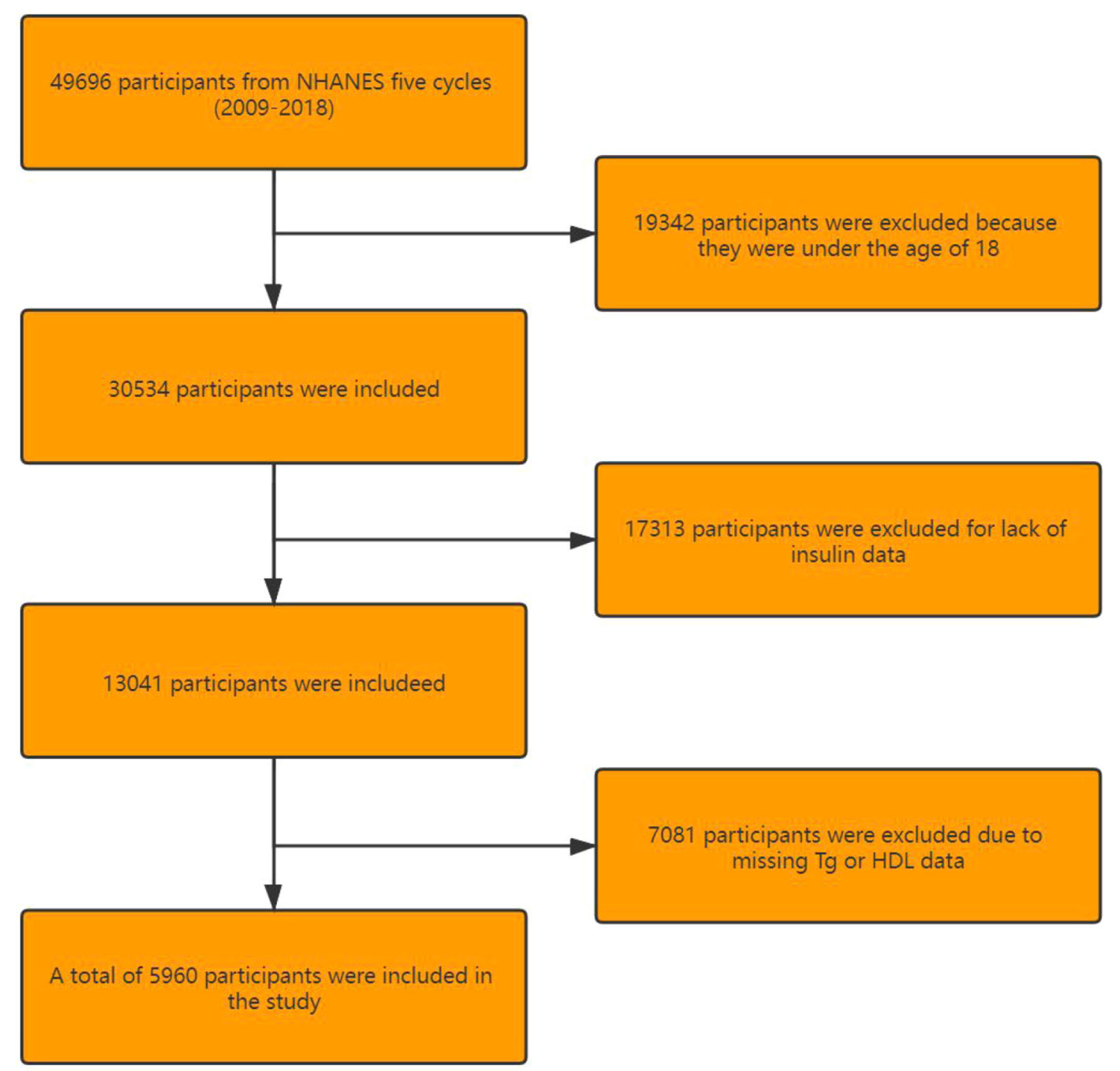

FIGURE 1 | Flowchart of participant selection.

mmol/L (type $1 \mathrm{DM}$, gestational DM, and specific types of DM were excluded) (21) (Figure 2).

\subsubsection{Hypertension}

In the present work, blood pressure (BP) was measured thrice while the participants were at rest, and the three measurements were averaged to assess whether the participants had hypertension or not. Typically, hypertension was defined as systolic BP $(\mathrm{SBP}) \geq 140 \mathrm{mmHg}$ and/or diastolic $\mathrm{BP}(\mathrm{DBP}) \geq 90$ $\mathrm{mmHg}$ or self-reported hypertension and use of antihypertensive drugs. The definition conformed to the American Heart Association Blood Pressure Guidelines 2017 (22).

\subsubsection{Smoking}

Participants were classified into three groups according to their different smoking conditions, namely, 1) current smokers (who smoked at least one cigarette a day in the past 30 days), 2) current non-smokers (who smoked an average of less than 1 cigarette per day in the past 30 days or more than 100 cigarettes in their lifetime), and 3) non-smokers (who reported that they smoked less than 100 cigarettes in their lifetime or never smoked). In this study, due to the small number of non-smokers, the current nonsmokers and non-smokers were finally combined as nonsmokers (23).

\subsubsection{Alcohol Use}

After the classification of alcohol consumption in previous studies was checked, the participants were finally divided into two groups, including drinkers (who drank more than 12 drinks a year) and non-drinkers (who drank no more than 12 drinks a year) (24). 
A

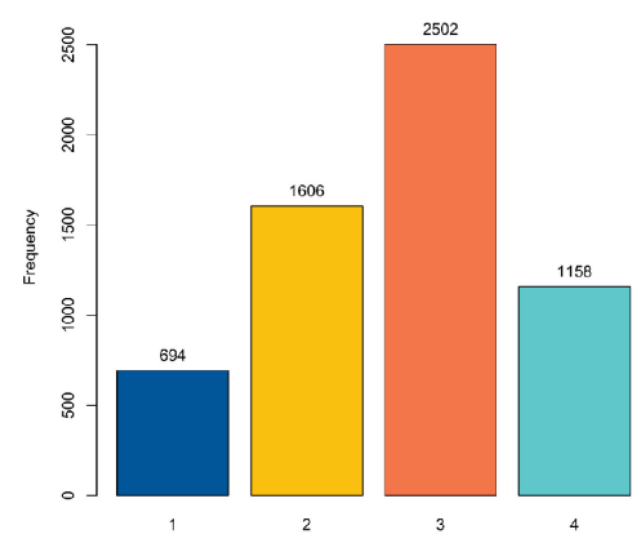

C

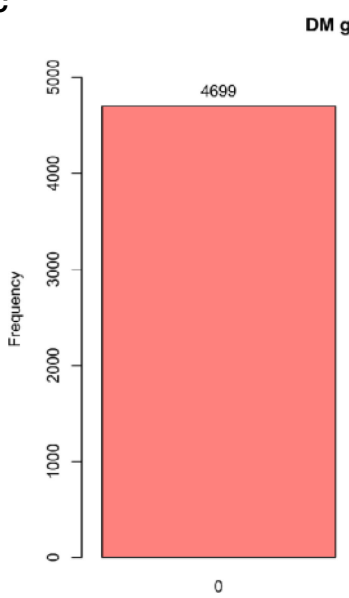

B

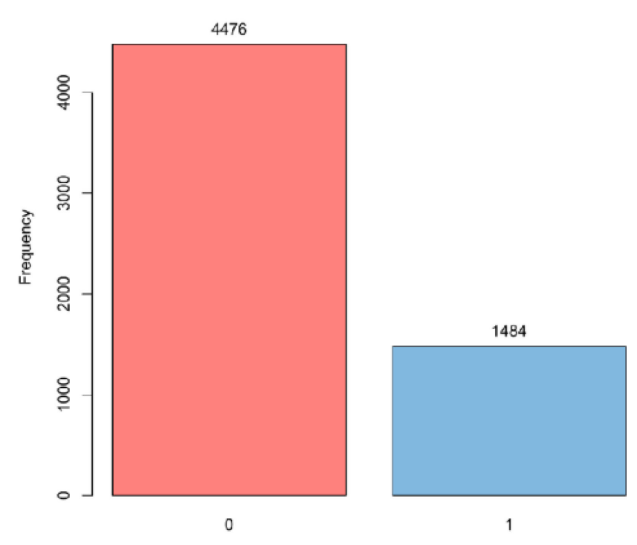

D

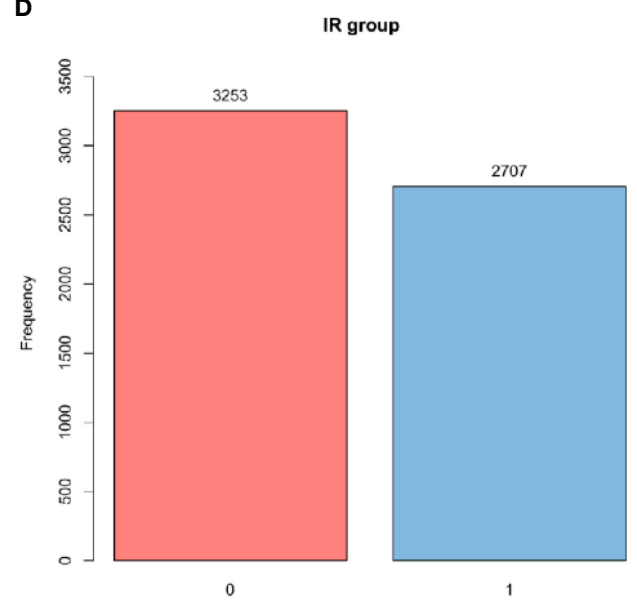

FIGURE 2 | Group diagram of each group. (A) Number of participants in different VD3 groups. (B) Number of participants with or without IGT. (C) Number of participants with or without DM. (D) Number of participants with or without IR. VD3, vitamin D3; IGT, impaired glucose tolerance; DM, diabetes mellitus; IR, insulin resistance.

\subsubsection{Income and Education}

In this study, participants earning more than $\$ 100,000$ were defined as high income, while those with a junior college education or higher were defined as high education.

\section{STATISTICAL ANALYSIS}

The NHANES database selects an annual of 5,000 people from a framework of 15 different locations in all counties of the United States to ensure that the data are universal and widespread. The unique multistage probability sampling technique employed by the NHANES database enables the data to better represent the incidence of IR in the U.S. population over the past few years. In this study, samples were collected from the NHANES database at five consecutive periods to make this study more convincing. Besides, data were selected through rigorous comprehensive screening to obtain a more representative sample.
Statistical analysis was performed using $\mathrm{R}$ language version 4.0.2, and a two-sided $p$-value $<0.05$ indicated statistical significance. Multivariate logistic regression was adopted to analyze the associations of TG/HDL ratio with IR, IGT, and DM, under different VD3 levels in the American adult population. Continuous variables were represented by detailed sample descriptions. Classification variables were expressed as counts and weighted percentages. In addition, the skewed distribution was based on the median and Q1-Q3, whereas the normal distribution was described by the median and SD. Four multivariate logistic regression models and smooth fitting curves were established to analyze the relationships of TG/HDL ratio with IR, IGT, and DM at different VD3 levels. Moreover, two-stage logistic regression and a log-likelihood ratio test were performed to analyze whether there was a non-linear relationship. Multiple imputations were utilized to compensate for the missing variables in this study. To increase the statistical power and avoid bias, the missing data of covariates were removed from this analysis. 
A sensitivity analysis was also performed to evaluate whether the generated data differed considerably from the raw data. According to sensitivity analysis, the generated complete data were similar to the raw data. As a result, our following multivariable analyses were carried out using the raw data based on Rubin's guidelines.

\subsection{Selection of Covariates}

In this study, covariates were screened according to the following criteria, 1) baseline characteristics of the population; 2) variables affecting TG/HDL, IR, IGT, and DM identified in previous studies; 3) the basic model changed by more than $10 \%$ after introducing covariates; and 4) experience gained in clinical work.

In line with the abovementioned criteria, gender, age, race, alanine aminotransferase (ALT), aspartate aminotransferase (AST), TG, total cholesterol (TC), $\gamma$-glutamyl transpeptidase (GGT), albumin (ALB), HDL, smoking, alcohol use, income, and education were selected as the covariates.

\section{RESULTS}

\subsection{Characteristics of the Participants}

A total of 5,960 participants were finally included according to the strict inclusion and exclusion criteria, including 2,929 males (49.1\%) and 3,031 females (50.9\%). Among these samples, there were significant differences in variables of gender, age, race, smoking, education, hyperuricemia, hypertension, hypercholesterolemia, body mass index (BMI), waist, IR, DM, IGT, ALT, AST, alkaline phosphatase (ALP), total bilirubin (TBIL), blood urea nitrogen (BUN), and GGT $(p<0.001)$ among VD3 groups $(\mathrm{N} 1-\mathrm{N} 4)$. Notably, the highest proportions of smoking (79.1\%), alcohol use (34.3\%), IR (55.6\%), IGT (31.7\%), and DM (26.9\%), together with the highest values of BMI $(30.6 \pm 8.8)$ and waist $(101.2 \pm 20.8)$ were observed in the $\mathrm{N} 1$ group. By contrast, the highest proportions of education $(61.3 \%)$, income $(45.3 \%)$, hyperuricemia $(23.1 \%)$, hypertension (77.0\%), and hypercholesterolemia (54.8\%), as well as the highest values of AST $(23.0,(20.0,27.0))$ and BUN (4.6, (3.9, $6.1)$ ), were seen in the $\mathrm{N} 4$ group. The average age of the total samples was $43.0 \pm 20.6$ years, and the oldest age $(52.8 \pm 19.9)$ was observed in the N4 group (Table 1).

\subsection{Univariate Logistic Analysis}

Table 2 presents the results of a univariate analysis to elucidate factors related to IR, IGT, and DM. As a result, the positive factors were age, drinking, hypertension, hyperuricemia, hypercholesterolemia, BMI, waist, TG, ALT, BUN, GGT, and TG/HDL ratio, whereas education, income, smoking, and HDL and VD3 levels were negatively correlated with IR, IGT, and DM (Table 2). Among the above variables, TG/HDL ratio showed the most significant relationship with IR among the diverse continuous variables, but its relations with IGT and DM were weaker (Table 2).

\subsection{Multiple Logistic Regression Models}

Four models were constructed to analyze the independent effect of the TG/HDL ratio on IR, IGT, and DM at different VD3 levels. The odds ratios (ORs) and 95\% CIs are shown in Table 3. The sizes of
ORs and 95\% CIs were interpreted as the corresponding changes in the incidence rates of IR, IGT, and DM with the increase in TG/ HDL ratio by one SD. In the fully adjusted model (Model 4 ), the ORs of the association between TG/HDL ratio and IR were 2.11 (1.57 2.83), 1.52 (1.29 1.78), 1.28 (1.16 1.41), and 2.16 $(1.75 \sim 2.67)$ in the N1-N4 groups, respectively. Meanwhile, the ORs regarding the association between TG/HDL ratio and IGT were 1.41 (1.17 1.70), 1.18 (1.07 1.29), 1.09 (1.02 1.16), and 1.22 (1.05 1.42) in the N1-N4 groups, respectively. The ORs of the association between TG/HDL ratio and DM were 1.31 (1.1 1.55), 1.19 (1.08 1.31), $1.13(1.06 \sim 1.20)$, and $1.22(1.05 \sim 1.42)$ in the N1$\mathrm{N} 4$ groups, respectively. In addition, TG/HDL ratio was also related to VD3 levels in IR, IGT, and DM ( $p$ for interaction <0.001) (Table 3 and Figure 3). The relatively strongest relationships of TG/HDL with IR, IGT, and DM were observed at the lowest and highest levels of VD3 groups (N1 and N4, Table 3).

\subsection{Non-Linear Relationships}

Here, we analyzed the non-linear relationships of TG/HDL ratio with IR, IGT, and DM at different VD3 levels. Using the fully adjusted Model 4, we discovered different relationships among VD3 groups. The correlation was fitted by logistic regression (Figure 4), and the non-linear relationship was approved by double segmentation (Table 4). In addition, $p<0.05$ was obtained upon the log-likelihood ratio test. Thus, two-stage logistic regression was conducted to accurately describe the relationships of TG/HDL ratio with IR, IGT, and DM at different VD3 levels (Figure 4 and Table 4).

\subsubsection{Insulin Resistance Group}

Based on the two-stage logistic regression models and recursive algorithm, the cutoff value was determined to be 1.094 . On the left side of the cutoff value, the ORs and 95\% CIs were 5.68 (2.6412.21), 5.55 (3.27-9.42), 3.50 (2.53-4.84), and 9.55 (3.79-24.06) in the N1-N4 groups, respectively. On the right side of the cutoff value, the ORs and 95\% CIs were $1.49(0.89,2.48), 1.41$ (1.03, $1.93), 1.54(1.12,2.11)$, and $1.84(1.33,2.54)$ in the $\mathrm{N} 1-\mathrm{N} 4$ groups, separately.

\subsubsection{Impaired Glucose Tolerance Group}

According to the two-stage logistic regression models and recursive algorithm, the cutoff value was set to 1.54 . On the left side of the cutoff value, the ORs and 95\% CIs were 5.29 (2.949.49), 2.68 (1.61-4.45), 2.14 (1.32-3.68), and 1.91 (1.40-2.61) in the N1-N4 groups, respectively. On the right side of the cutoff value, the ORs and 95\% CIs were $1.13(0.63,2.01), 1.2(0.85$, $1.68), 1.40(1.1,1.71)$, and $0.06(0.01,0.46)$ in the $\mathrm{N} 1-\mathrm{N} 4$ groups, separately.

\subsubsection{Diabetes Mellitus Group}

In line with the two-stage logistic regression models and recursive algorithm, the cutoff value was 1.11. On the left side of the cutoff value, the ORs and 95\% CIs were 7.33 (2.43-22.05), 3.83 (1.04$14.18), 7.13$ (2.30-22.08), and $1.33(0.99-1.77)$ in the N1-N4 groups, respectively. On the right side of the cutoff value, the ORs and $95 \%$ CIs were $1.20(0.77,1.85), 1.53(1.19,1.97), 1.57$ (1.29, $1.94)$, and $0.54(0.11,2.65)$ in the $\mathrm{N} 1-\mathrm{N} 4$ groups, respectively. 
TABLE 1 | Basic characteristics of the participants.

\begin{tabular}{|c|c|c|c|c|c|c|}
\hline Variables & Total $(n=5,960)$ & N1 (n = 694) & N2 $(n=1,606)$ & N3 (n= 2,502) & N4 ( $n=1,158)$ & p-Value \\
\hline Gender, n (\%) & & & & & & $<0.001$ \\
\hline Male & $2,929(49.1)$ & $305(43.9)$ & $786(48.9)$ & $1,376(55)$ & $462(39.9)$ & \\
\hline Female & 3,031 (50.9) & $389(56.1)$ & $820(51.1)$ & $1,126(45)$ & $696(60.1)$ & \\
\hline Age (mean \pm SD) & $43.0 \pm 20.6$ & $41.2 \pm 18.9$ & $38.5 \pm 19.3$ & $41.9 \pm 20.7$ & $52.8 \pm 19.9$ & $<0.001$ \\
\hline Race (n (\%)) & & & & & & $<0.001$ \\
\hline Mexican American & $804(13.5)$ & 89 (12.8) & $299(18.6)$ & $350(14)$ & $66(5.7)$ & \\
\hline Other Hispanics & $576(9.7)$ & $36(5.2)$ & $156(9.7)$ & $297(11.9)$ & $87(7.5)$ & \\
\hline Non-Hispanic white & 2,331 (39.1) & $116(16.7)$ & 357 (22.2) & 1,096 (43.8) & $762(65.8)$ & \\
\hline Non-Hispanic black & $1,311(22.0)$ & $342(49.3)$ & 497 (30.9) & $370(14.8)$ & $102(8.8)$ & \\
\hline Other race & $938(15.7)$ & $111(16)$ & $297(18.5)$ & $389(15.5)$ & $141(12.2)$ & \\
\hline Alcohol use (n (\%)) & & & & & & 0.095 \\
\hline No & 4,113 (69.0) & $456(65.7)$ & 1,082 (67.4) & $1,762(70.4)$ & $813(70.2)$ & \\
\hline Yes & 1,842 (30.9) & 238 (34.3) & $522(32.5)$ & $738(29.5)$ & $344(29.7)$ & \\
\hline SMOK (n (\%)) & & & & & & $<0.001$ \\
\hline No & 1,786 (30.0) & $145(20.9)$ & $448(27.9)$ & $761(30.4)$ & $432(37.3)$ & \\
\hline Yes & $4,174(70.0)$ & $549(79.1)$ & $1,158(72.1)$ & $1,741(69.6)$ & $726(62.7)$ & \\
\hline EDU (n (\%)) & & & & & & $<0.001$ \\
\hline No higher education & $2,668(44.8)$ & $339(48.8)$ & $749(46.6)$ & $1,132(45.2)$ & $448(38.7)$ & \\
\hline Higher education & $3,292(55.2)$ & $355(51.2)$ & $857(53.4)$ & $1,370(54.8)$ & $710(61.3)$ & \\
\hline INCOME (n (\%)) & & & & & & $<0.001$ \\
\hline No more than $\$ 100,000$ & 3,657 (61.4) & 495 (71.3) & 1,037 (64.6) & 1,492 (59.6) & $633(54.7)$ & \\
\hline More than $\$ 100,000$ & $2,303(38.6)$ & $199(28.7)$ & $569(35.4)$ & $1,010(40.4)$ & $525(45.3)$ & \\
\hline HUA (n (\%)) & & & & & & $<0.001$ \\
\hline No & 4,849 (81.4) & $536(77.2)$ & 1,307 (81.4) & 2,116 (84.6) & $890(76.9)$ & \\
\hline Yes & $1,111(18.6)$ & $158(22.8)$ & $299(18.6)$ & $386(15.4)$ & $268(23.1)$ & \\
\hline Hbp (n (\%)) & & & & & & $<0.001$ \\
\hline No & $1,889(31.7)$ & $181(26.1)$ & $581(36.2)$ & $861(34.4)$ & $266(23)$ & \\
\hline Yes & 4,071 (68.3) & $513(73.9)$ & $1,025(63.8)$ & $1,641(65.6)$ & $892(77)$ & \\
\hline HTC (n (\%)) & & & & & & $<0.001$ \\
\hline No & $3,405(57.1)$ & $408(58.8)$ & 1,024 (63.8) & $1,450(58)$ & $523(45.2)$ & \\
\hline Yes & $2,555(42.9)$ & $286(41.2)$ & $582(36.2)$ & $1,052(42)$ & $635(54.8)$ & \\
\hline BMI, (mean \pm SD) & $28.0 \pm 7.1$ & $30.6 \pm 8.8$ & $28.5 \pm 7.4$ & $27.4 \pm 6.5$ & $27.1 \pm 6.3$ & $<0.001$ \\
\hline Waist (mean \pm SD) & $95.8 \pm 17.7$ & $101.2 \pm 20.8$ & $96.1 \pm 18.2$ & $94.5 \pm 16.9$ & $94.8 \pm 15.8$ & $<0.001$ \\
\hline Insulin resistance (n (\%)) & & & & & & $<0.001$ \\
\hline No & 3,253 (54.6) & $308(44.4)$ & $780(48.6)$ & 1,407 (56.2) & $758(65.5)$ & \\
\hline Yes & 2,707 (45.4) & $386(55.6)$ & $826(51.4)$ & 1,095 (43.8) & $400(34.5)$ & \\
\hline Diabetes (n (\%)) & & & & & & $<0.001$ \\
\hline No & 4,699 (78.8) & $507(73.1)$ & 1,257 (78.3) & 2,036 (81.4) & $899(77.6)$ & \\
\hline Yes & $1,261(21.2)$ & $187(26.9)$ & $349(21.7)$ & 466 (18.6) & $259(22.4)$ & \\
\hline Prediabetes (n (\%)) & & & & & & $<0.001$ \\
\hline No & $4,476(75.1)$ & $474(68.3)$ & $1,211(75.4)$ & $1,945(77.7)$ & $846(73.1)$ & \\
\hline Yes & $1,484(24.9)$ & $220(31.7)$ & $395(24.6)$ & 557 (22.3) & $312(26.9)$ & \\
\hline ALT (median (IQR)) & $19.0(15.0,26.0)$ & $19.0(14.0,25.0)$ & $19.0(15.0,27.0)$ & $20.0(15.0,27.0)$ & $20.0(16.0,25.0)$ & 0.017 \\
\hline AST (median (IQR)) & $22.0(19.0,27.0)$ & $21.0(18.0,26.0)$ & $22.0(19.0,26.0)$ & $22.0(19.0,27.0)$ & $23.0(20.0,27.0)$ & $<0.001$ \\
\hline ALP (median (IQR)) & $66.0(54.0,84.0)$ & $68.0(55.0,85.0)$ & $68.0(55.0,87.0)$ & $66.0(54.0,85.0)$ & $63.0(51.0,77.0)$ & $<0.001$ \\
\hline TBIL (median (IQR)) & $12.0(8.6,13.7)$ & $10.3(8.6,13.7)$ & $10.3(8.6,13.7)$ & $12.0(8.6,13.7)$ & $12.0(8.6,13.7)$ & $<0.001$ \\
\hline BUN (median (IQR)) & $4.3(3.2,5.4)$ & $3.6(2.9,4.6)$ & $3.9(3.2,4.6)$ & $4.3(3.6,5.4)$ & $4.6(3.9,6.1)$ & $<0.001$ \\
\hline TC (median (IQR)) & $4.7(4.0,5.4)$ & $4.6(4.0,5.4)$ & $4.6(4.0,5.4)$ & $4.6(4.0,5.4)$ & $4.9(4.2,5.6)$ & $<0.001$ \\
\hline UA (median (IQR)) & $315.2(261.7,374.7)$ & $324.2(255.8,386.6)$ & $315.2(255.8,374.7)$ & $315.2(261.7,368.8)$ & $309.3(261.7,374.7)$ & 0.426 \\
\hline GGT (median (IQR)) & $17.0(13.0,26.0)$ & $18.0(13.2,31.0)$ & $18.0(13.0,27.0)$ & $17.0(12.0,25.0)$ & $17.0(13.0,26.0)$ & $<0.001$ \\
\hline FPG (median (IQR)) & $5.2(4.8,5.7)$ & $5.2(4.8,5.8)$ & $5.2(4.8,5.7)$ & $5.2(4.8,5.6)$ & $5.2(4.8,5.7)$ & 0.092 \\
\hline TG/HDL (median (IQR)) & $0.8(0.5,1.3)$ & $0.8(0.5,1.3)$ & $0.7(0.5,1.3)$ & $0.8(0.5,1.4)$ & $0.8(0.5,1.3)$ & 0.071 \\
\hline
\end{tabular}

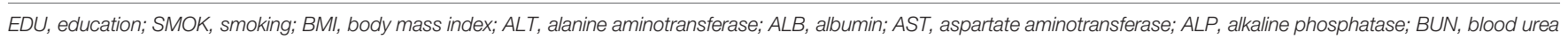
nitrogen; GGT, r-glutamyl transpeptidase; UA, uric acid; FPG, fasting plasma glucose; TC, total cholesterol; TG/HDL, triglycerides/high-density lipoprotein.

\section{DISCUSSION}

In this study, 5,960 participants were recruited to analyze the independent associations of TG/HDL ratio with IR, IGT, and DM at different VD3 levels. After the influencing factors were adjusted, TG/HDL ratio was related to IR, IGT, and DM at varying degrees at different VD3 levels ( $p$ for interaction $<0.05$ ). Specifically, stronger relationships were observed in the N1 and
N4 groups (Table 3). It suggests that the too low or too high VD3 levels possibly strengthen the associations of TG/HDL ratio with IR, IGT, and DM.

In a study carried out on Hispanic and African Americans, the associations of TG/HDL ratio with IR, $\beta$-cell function, and DM were investigated. After the influencing factors were adjusted, TG/HDL ratio was associated with IR in the nonCaucasian populations, and a higher TG/HDL ratio was related 
TABLE 2 | Univariate analysis of IR, IGT, and DM.

\begin{tabular}{|c|c|c|c|c|c|c|}
\hline \multirow[t]{2}{*}{ Variables } & \multicolumn{2}{|c|}{ IR } & \multicolumn{2}{|c|}{ IGT } & \multicolumn{2}{|c|}{ DM } \\
\hline & OR (95\% Cl) & p-Value & OR (95\% Cl) & p-Value & OR (95\% Cl) & p-Value \\
\hline Age & $1.01(1 \sim 1.01)$ & $<0.001$ & $1.06(1.05 \sim 1.06)$ & $<0.001$ & $1.06(1.06 \sim 1.06)$ & $<0.001$ \\
\hline \multicolumn{7}{|l|}{ Gender } \\
\hline Male & 1 & & 1 & & 1 & \\
\hline Female & $0.99(0.9 \sim 1.1)$ & 0.89 & $0.79(0.7 \sim 0.89)$ & $<0.001$ & $0.85(0.75 \sim 0.96)$ & 0.011 \\
\hline \multicolumn{7}{|l|}{ Education } \\
\hline No higher education & 1 & & 1 & & 1 & \\
\hline Higher education & $0.8(0.72 \sim 0.89)$ & $<0.001$ & $0.65(0.58 \sim 0.74)$ & $<0.001$ & 0.62 (0.55 0.7) & $<0.001$ \\
\hline \multicolumn{7}{|l|}{ Income } \\
\hline No more than $\$ 100,000$ & 1 & & 1 & & 1 & \\
\hline More than $\$ 100,000$ & $0.75(0.68 \sim 0.84)$ & $<0.001$ & $0.74(0.66 \sim 0.84)$ & $<0.001$ & $0.72(0.63 \sim 0.82)$ & $<0.001$ \\
\hline \multicolumn{7}{|l|}{ Alcohol use } \\
\hline No & 1 & & 1 & & 1 & \\
\hline Yes & $1.25(1.12 \sim 1.4)$ & $<0.001$ & $1.15(1.02 \sim 1.31)$ & 0.028 & $1.22(1.07 \sim 1.39)$ & 0.003 \\
\hline \multicolumn{7}{|l|}{ Smoking } \\
\hline No & 1 & & 1 & & 1 & \\
\hline Yes & $0.93(0.83 \sim 1.04)$ & 0.195 & $0.74(0.65 \sim 0.84)$ & $<0.001$ & $0.76(0.67 \sim 0.87)$ & $<0.001$ \\
\hline \multicolumn{7}{|l|}{ Hyperuricemia } \\
\hline No & 1 & & 1 & & 1 & \\
\hline Yes & 2.6 (2.27 2.98) & $<0.001$ & $2.43(2.11 \sim 2.79)$ & $<0.001$ & $2.32(2.01 \sim 2.67)$ & $<0.001$ \\
\hline \multicolumn{7}{|l|}{ Hypertension } \\
\hline No & 1 & & 1 & & 1 & \\
\hline Yes & $1.71(1.53 \sim 1.91)$ & $<0.001$ & $6.56(5.43 \sim 7.91)$ & $<0.001$ & 7.58 (6.1 9.42) & $<0.001$ \\
\hline \multicolumn{7}{|l|}{ Hypercholesterolemia } \\
\hline No & 1 & & 1 & & 1 & \\
\hline Yes & $1.73(1.56 \sim 1.92)$ & $<0.001$ & $4.14(3.65 \sim 4.7)$ & $<0.001$ & $4.48(3.91 \sim 5.13)$ & $<0.001$ \\
\hline BMl & 1.18 (1.17 1.2) & $<0.001$ & $1.1(1.09 \sim 1.11)$ & $<0.001$ & 1.09 (1.08 1.1) & $<0.001$ \\
\hline Waist & 1.07 (1.06 1.07) & $<0.001$ & 1.05 (1.05 1.06) & $<0.001$ & 1.05 (1.05 1.05) & $<0.001$ \\
\hline TC & $1.04(0.99 \sim 1.09)$ & 0.097 & $1.13(1.07 \sim 1.19)$ & $<0.001$ & $1.11(1.05 \sim 1.17)$ & $<0.001$ \\
\hline TG & $2.06(1.91 \sim 2.21)$ & $<0.001$ & $1.48(1.39 \sim 1.57)$ & $<0.001$ & $1.44(1.36 \sim 1.53)$ & $<0.001$ \\
\hline HDL & $0.15(0.13 \sim 0.18)$ & $<0.001$ & $0.41(0.35 \sim 0.49)$ & $<0.001$ & $0.41(0.34 \sim 0.49)$ & $<0.001$ \\
\hline TG/HDL & 2.09 (1.94 2.25) & $<0.001$ & $1.3(1.24 \sim 1.36)$ & $<0.001$ & $1.28(1.22 \sim 1.34)$ & $<0.001$ \\
\hline ALT & $1.02(1.02 \sim 1.02)$ & $<0.001$ & $1.01(1.01 \sim 1.01)$ & $<0.001$ & $1.01(1.01 \sim 1.01)$ & $<0.001$ \\
\hline AST & $1(1 \sim 1.01)$ & 0.174 & $1(1 \sim 1.01)$ & 0.005 & $1(1 \sim 1.01)$ & 0.032 \\
\hline GGT & $1.01(1.01 \sim 1.01)$ & $<0.001$ & $1.01(1.01 \sim 1.01)$ & $<0.001$ & $1.01(1.01 \sim 1.01)$ & $<0.001$ \\
\hline ALP & $1(1 \sim 1)$ & $<0.001$ & $1(1 \sim 1)$ & $<0.001$ & $1(1 \sim 1)$ & $<0.001$ \\
\hline BUN & $1.03(1.01 \sim 1.06)$ & 0.009 & 1.32 (1.27 1.36) & $<0.001$ & 1.32 (1.27 1.36) & $<0.001$ \\
\hline UA & $1.01(1 \sim 1.01)$ & $<0.001$ & 1.01 (1 1.01) & $<0.001$ & $1.01(1 \sim 1.01)$ & $<0.001$ \\
\hline
\end{tabular}

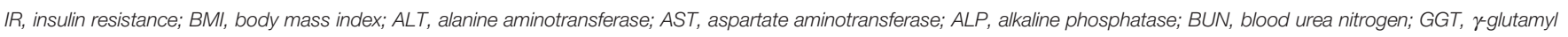
transpeptidase; UA, uric acid; TC, total cholesterol; TG, triglycerides; HDL, high-density lipoprotein.

to lower insulin sensitivity in the Hispanic and African American populations (25). Wang et al. also confirmed that TG/HDL ratio was an independent risk factor for DM in the Singapore Chinese (26). In addition, Gong et al. studied more than 100,000 Chinese cohorts and discovered that a higher TG/HDL ratio was positively correlated with the occurrence of IGT and DM after the influencing factors were adjusted (27). These investigations are consistent with our results.

This study further evaluated the effect of VD3 levels on the relationships of TG/HDL ratio with IR, IGT, and DM. As a result, too high or too low VD3 levels promoted the abovementioned relationships. The N3 group (ordinary VD3 levels) showed the weakest correlations of TG/HDL with IR, IGT, and DM. This indicates that, compared with VD3 abundance and deficiency, maintaining the reasonable VD3 levels is more effective in reducing the associations of TG/HDL ratio with IR, IGT, and DM. In addition, we found that such associations were strengthened, not weakened as expected, in the N4 group. This demonstrates that excessive (high level of) VD3 strengthens the associations of TG/ HDL with IR, IGT, and DM. The main reason is that excessive VD3 can lead to abnormalities in calcium and phosphorus metabolism as well as VD3 toxicity in the body, thus lowering the protective effect of VD3 on the body. Therefore, this should be further validated by more clinical studies and randomized controlled trials (RCTs).

As reported in previous studies, high TG/HDL ratios lead to reduced retention of fatty acids; therefore, more fatty acids are transported to the liver for TG synthesis, and more free fatty acids will be formed accordingly (28), while higher free fatty acid levels have been identified as a risk factor for T2DM. By combining with insulin, the excess free fatty acids prevent the secretion of a normal amount of insulin from achieving the desired glucose-lowering effect. As a result, pancreatic $\beta$-cells are stimulated to secrete more insulin, which thus leads to IR and ultimately the development of DM (29). In addition, TG-rich lipoproteins accelerate the production of leptin, angiotensinogen, tumor necrosis factor-alpha (TNF-a), interleukin 6 (IL-6), 
TABLE 3 | Multiple regression analysis of different VD3 levels.

\begin{tabular}{|c|c|c|c|c|c|c|c|c|c|}
\hline \multirow[t]{2}{*}{ Outcomes } & \multicolumn{2}{|c|}{ Model 1} & \multicolumn{2}{|c|}{ Model 2} & \multicolumn{2}{|c|}{ Model 3} & \multicolumn{2}{|c|}{ Model 4} & \multirow[t]{2}{*}{$p$ for interaction } \\
\hline & OR (95\% Cl) & p-Value & OR (95\% Cl) & p-Value & OR (95\% Cl) & p-Value & OR (95\% Cl) & p-Value & \\
\hline IR group & & & & & & & & & $<0.001$ \\
\hline $\mathrm{N} 1(<30)$ & $2.33(1.83 \sim 2.96)$ & $<0.001$ & 2.63 (2.02 3.43) & $<0.001$ & 2.02 (1.55 2.64) & $<0.001$ & $2.11(1.57 \sim 2.83)$ & $<0.001$ & \\
\hline N2 (30-50) & $2.01(1.73 \sim 2.34)$ & $<0.001$ & 2.23 (1.89 2.63) & $<0.001$ & $1.52(1.31 \sim 1.78)$ & $<0.001$ & $1.52(1.29 \sim 1.78)$ & $<0.001$ & \\
\hline N3 (50-80) & 1.87 (1.68 2.07) & $<0.001$ & 1.88 (1.69 2.09) & $<0.001$ & $1.31(1.18 \sim 1.44)$ & $<0.001$ & $1.28(1.16 \sim 1.41)$ & $<0.001$ & \\
\hline N4 (>80) & 3.27 (2.69 3.98) & $<0.001$ & 3.22 (2.63 3.94) & $<0.001$ & $2.23(1.81 \sim 2.74)$ & $<0.001$ & $2.16(1.75 \sim 2.67)$ & $<0.001$ & \\
\hline IGT group & & & & & & & & & $<0.001$ \\
\hline $\mathrm{N} 1(<30)$ & $1.54(1.33 \sim 1.8)$ & $<0.001$ & 1.63 (1.36 1.94) & $<0.001$ & $1.46(1.21 \sim 1.75)$ & $<0.001$ & $1.41(1.17 \sim 1.70)$ & $<0.001$ & \\
\hline N2 (30-50) & $1.31(1.19 \sim 1.43)$ & $<0.001$ & $1.29(1.17 \sim 1.43)$ & $<0.001$ & $1.18(1.08 \sim 1.3)$ & $<0.001$ & $1.18(1.07 \sim 1.29)$ & 0.001 & \\
\hline N3 (50-80) & $1.18(1.11 \sim 1.26)$ & $<0.001$ & $1.19(1.1 \sim 1.27)$ & $<0.001$ & 1.08 (1.01 1.15) & 0.016 & 1.09 (1.02 1.16) & 0.007 & \\
\hline N4 $(>80)$ & $1.6(1.39 \sim 1.84)$ & $<0.001$ & 1.59 (1.37 1.85) & $<0.001$ & $1.28(1.1 \sim 1.48)$ & 0.001 & $1.22(1.05 \sim 1.42)$ & 0.009 & \\
\hline DM group & & & & & & & & & $<0.001$ \\
\hline $\mathrm{N} 1(<30)$ & $1.43(1.24 \sim 1.65)$ & $<0.001$ & $1.51(1.27 \sim 1.78)$ & $<0.001$ & $1.37(1.15 \sim 1.62)$ & $<0.001$ & $1.31(1.1 \sim 1.55)$ & 0.002 & \\
\hline N2 (30-50) & $1.29(1.18 \sim 1.41)$ & $<0.001$ & $1.31(1.19 \sim 1.44)$ & $<0.001$ & $1.2(1.09 \sim 1.32)$ & $<0.001$ & 1.19 (1.08 1.31) & $<0.001$ & \\
\hline N3 (50-80) & $1.18(1.1 \sim 1.26)$ & $<0.001$ & $1.22(1.13 \sim 1.32)$ & $<0.001$ & $1.11(1.04 \sim 1.2)$ & 0.002 & $1.13(1.06 \sim 1.20)$ & $<0.001$ & \\
\hline N4 $(>80)$ & $1.51(1.32 \sim 1.73)$ & $<0.001$ & $1.53(1.31 \sim 1.78)$ & $<0.001$ & $1.27(1.09 \sim 1.47)$ & 0.002 & $1.22(1.05 \sim 1.42)$ & 0.01 & \\
\hline
\end{tabular}

Model 1: non-adjusted. Model 2: adjusted for gender, age, and race. Model 3: Model $2+$ adjusted for ALT, AST, TG, TC, GGT, ALB, and HDL. Model 4: Model $3+$ adjusted for smoking, alcohol use, income, and education.

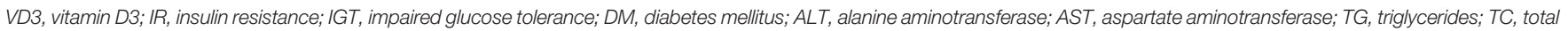
cholesterol; GGT, rglutamyl transpeptidase; ALB, albumin; HDL, high-density lipoprotein.

fibrinogen activator inhibitor 1, transforming growth factor B (TGF-b), lipocalin, and resistin (29). These factors are the risk factors for the development of IR or DM, at least at the experimental level. VD3 can bind to some free fatty acids (30) to alleviate certain unfavorable effects of fatty acids. This was also represented in results from the N1-N3 groups, where the associations of TG/HDL ratio with IR, IGT, and DM were weakened as VD3 levels increased (Tables 3 and 4). As a kind of fat-soluble vitamin, VD3 is mainly obtained by irradiating the skin with sunlight (31). Studies have reported that phototherapy and VD3 supplementation can ameliorate IR and inflammation in a rat model of non-alcoholic fatty liver disease (NAFLD) induced by a special diet (32). Based on these results and our observation, we suppose that VD3 is beneficial to normal metabolism, and safe sunlight exposure can be an appropriate way to increase VD3 levels for human health (33).

Certainly, some of the results in this study were different from those of previous studies. Such discrepancies may be explained from the following aspects. 1) The study populations were different. 2) The adjusted variables were different, and a more adequate adjustment strategy was adopted in this study. 3) The associations of TG/HDL ratio with IR, IGT, and DM were analyzed at different VD3 levels in this study. Notably, the clinical strength of this study is as follows. We found that within a reasonable range of VD3 (0$80 \mathrm{ng} / \mathrm{ml}$ ), increasing VD3 levels attenuated the associations of TG/ HDL ratio with IR, IGT, and DM. This provides new evidence for clinical guidance on the appropriate range of VD3 levels in patients. Meanwhile, we discovered that when VD3 levels were

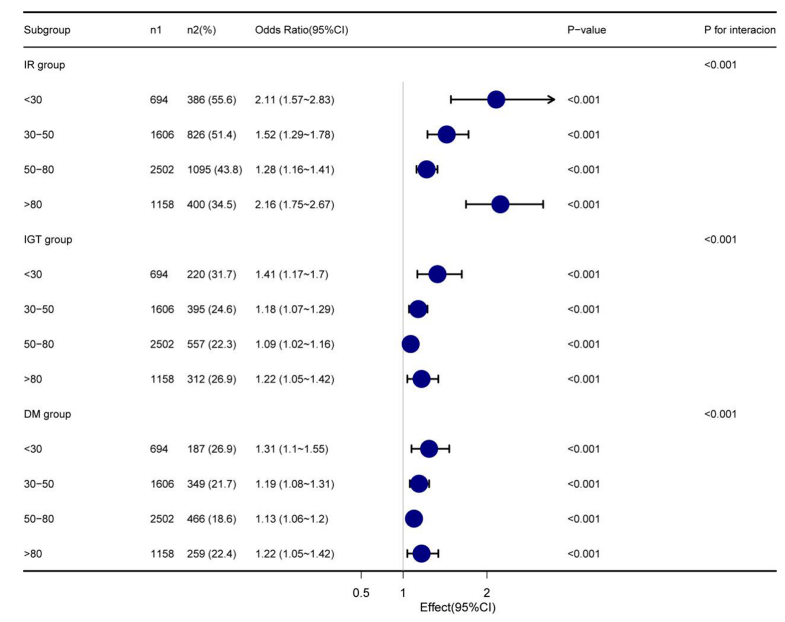

FIGURE 3 | Subgroup analysis based on the multivariate logistic regression analysis of the association between TG/HDL ratio and IR, IGT, and DM. TG/HDL, triglycerides/high-density lipoprotein; IR, insulin resistance; IGT, impaired glucose tolerance; DM, diabetes mellitus. 


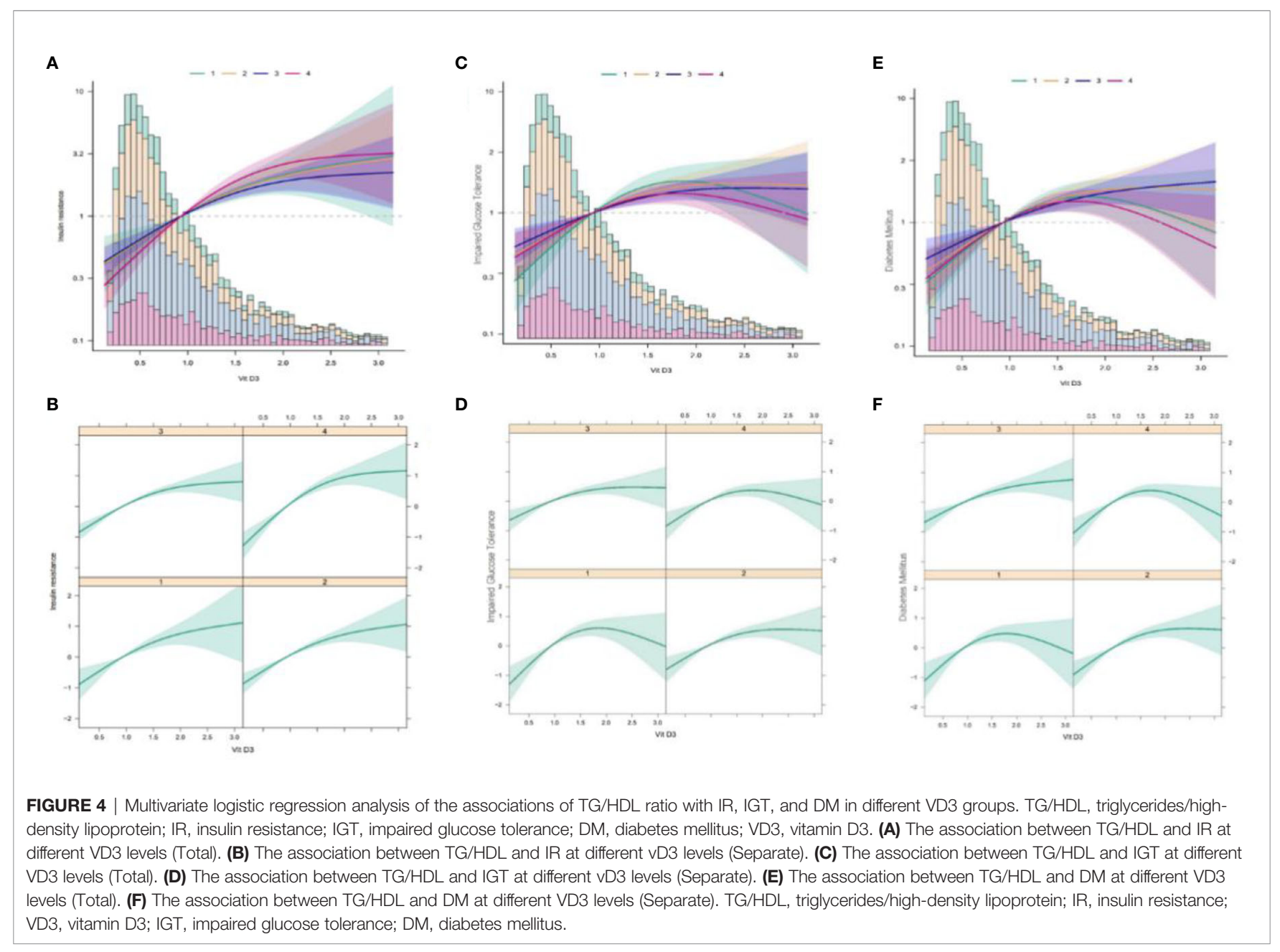

TABLE 4 | Threshold analysis of TG/HDL on the incident of IR, IGT, and DM in the NHANES study, 2009-2018.

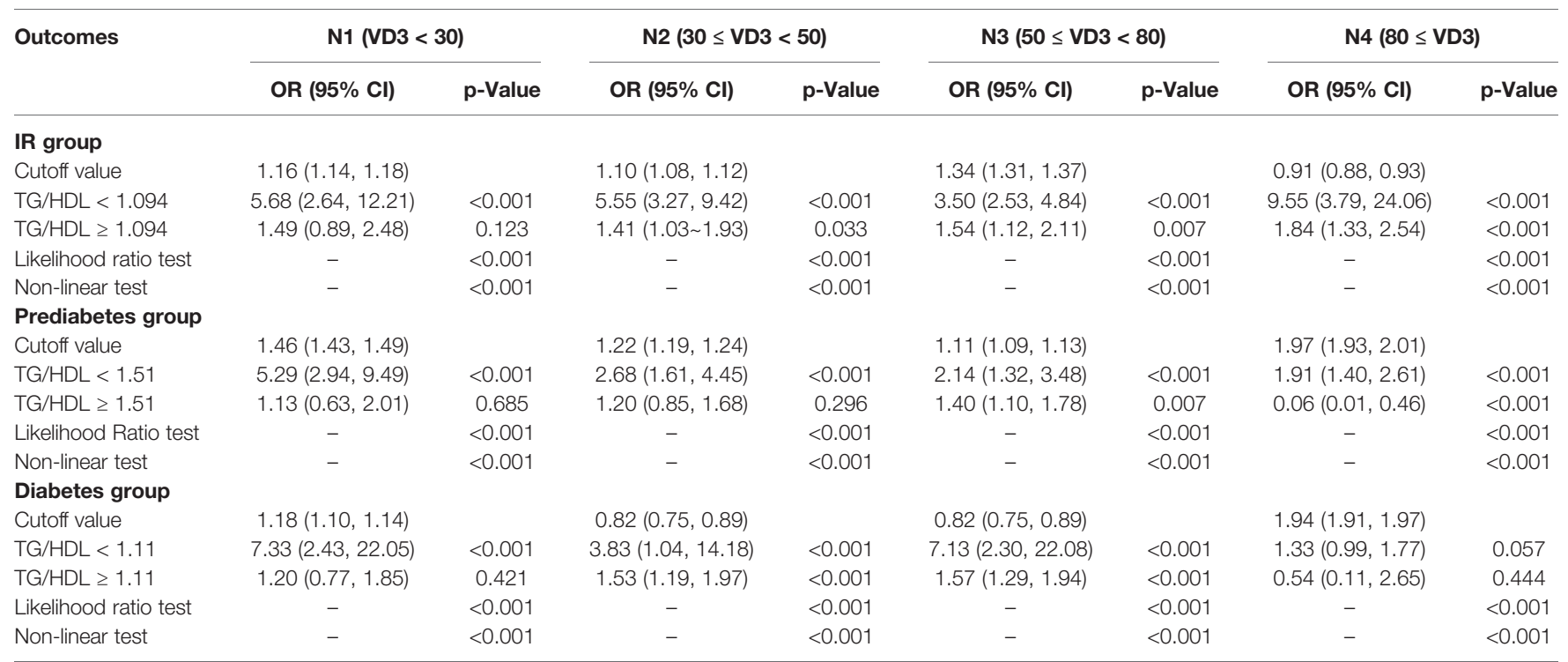

Adjusted for age, gender, race, education, BMI, WC (waist circumference), income, smoking, alcohol use, hypertension, UA, ALT, AST, GGT, LDH, and BUN.

TG/HDL, triglycerides/high-density lipoprotein; IR, insulin resistance; IGT, impaired glucose tolerance; DM, diabetes mellitus; VD3, vitamin D3; BMI, body mass index; UA, uric acid; ALT, alanine aminotransferase; AST, aspartate aminotransferase; GGT, r-glutamyl transpeptidase; LDH, low-density lipoprotein; BUN, blood urea nitrogen. 
too high $(\geq 80 \mathrm{ng} / \mathrm{ml})$ in $\mathrm{DM}$ patients, it might strengthen the relations of TG/HDL ratio with IR, IGT, and DM. This indicates that the incidence of abnormal glucose metabolism may increase in people with high TG/HDL ratios when VD3 levels are too high.

Nevertheless, certain limitations should also be noted in this study. 1) This was a cross-sectional study, and the causal relationships of TG/HDL ratio with IR, IGT, and DM were not determined. Therefore, a cohort study is warranted to analyze the accurate relationship. 2) Special populations (like pregnant women and children) were excluded from this study, and whether the results were applicable to these populations remains unknown. Also, there are some noteworthy highlights of this study. First, this study was conducted using the official NHANES database, which is more representative of the entire US population after a complex weighting design. Second, in the design of this study, smoothed fitting curve and two-stage logistic regression were used to accurately analyze the relationships.

\section{CONCLUSION}

Collectively, our results suggest that in the American population, maintaining too high or too low levels of VD3 can promote the associations of TG/HDL with IR, IGT, and DM, which shed new light on DM research. However, other conditions such as age and sunlight exposure level should be taken into consideration when formulating the appropriate VD3 levels.

\section{REFERENCES}

1. Kulda V. Vitamin D Metabolism. Vnitr Lek (2012) 58(5):400-4.

2. Baeke F, Takiishi T, Korf H, Gysemans C, Mathieu C. Vitamin D: Modulator of the Immune System. Curr Opin Pharmacol (2010) 10(4):482-96. doi: 10.1016/j.coph.2010.04.001

3. Wenclewska S, Szymczak-Pajor I, Drzewoski J, Bunk M, Śliwińska A. Vitamin D Supplementation Reduces Both Oxidative DNA Damage and Insulin Resistance in the Elderly With Metabolic Disorders. Int J Mol Sci (2019) 20 (12):2891-907. doi: 10.3390/ijms20122891

4. Lu L, Lu Q, Chen W, Li J, Li C, Zheng Z. Vitamin D(3) Protects Against Diabetic Retinopathy by Inhibiting High-Glucose-Induced Activation of the ROS/TXNIP/NLRP3 Inflammasome Pathway. J Diabetes Res (2018) 2018:8193523. doi: 10.1155/2018/8193523

5. Gong R, Xu Z, Wei X. The Association Between Vitamin D3 and Diabetes in Both Hyperuricemia and Non-Hyperuricemia Populations. Endocrine (2021) 74(1):90-9. doi: 10.1007/s12020-021-02778-y

6. Yang M, Xu J, Yu J, Yang B, Gan H, Li S, et al. Anti-Inflammatory Effects of 1,25-Dihydroxyvitamin D3 in Monocytes Cultured in Serum From Patients With Type 2 Diabetes Mellitus and Diabetic Nephropathy With Uremia via Toll-Like Receptor 4 and Nuclear Factor-אb P65. Mol Med Rep (2015) 12 (6):8215-22. doi: 10.3892/mmr.2015.4482

7. Kendall DM, Sobel BE, Coulston AM, Peters Harmel AL, McLean BK, Peragallo-Dittko V, et al. The Insulin Resistance Syndrome and Coronary Artery Disease. Coron Artery Dis (2003) 14(4):335-48. doi: 10.1097/ 01.mca.0000076512.29238.2a

8. Zhang XY, Guo CC, Yu YX, Xie L, Chang CQ. Establishment of High-Fat Diet-Induced Obesity and Insulin Resistance Model in Rats. Beijing Da Xue Xue Bao Yi Xue Ban (2020) 52(3):557-63. doi: 10.19723/j.issn.1671167X.2020.03.024

9. American Diabetes Association. Diagnosis and Classification of Diabetes Mellitus. Diabetes Care (2011) 34(Suppl 1):S62-9. doi: 10.2337/dc11-S062

\section{DATA AVAILABILITY STATEMENT}

Publicly available datasets were analyzed in this study. Data can be obtained from the NHANES database (https://www.cdc.gov/ nchs/nhanes/).

\section{AUTHOR CONTRIBUTIONS}

YL and RG: conceived the idea; YL, RG and GL wrote the manuscript; GL and RG collected and read the literature and revised the article; XW and LY read through and corrected the manuscript. All authors contributed to the article and approved the submitted version. YL is the first author. RG and GL are the co-first author. XW and LY are the corresponding author of this paper.

\section{FUNDING}

This work was supported by Grants from the National Key R\&D Program of China (2018YFC1311500), the National Natural Science Foundation of China (81860370), the CAS "Light of West China" Program (2019), the General Project of Natural Science Foundation of Qinghai Province (2020-ZJ-930) Effects of high altitude environment and season on vitamin $\mathrm{D}$, bone metabolism factors and muscle volume in adults.

10. Robert AA, Al Dawish MA. The Worrying Trend of Diabetes Mellitus in Saudi Arabia: An Urgent Call to Action. Curr Diabetes Rev (2020) 16(3):20410. doi: 10.2174/1573399815666190531093735

11. Holt RIG. Diabetes Education, Education and Education. Diabetes Med (2017) 34(8):1023-4. doi: 10.1111/dme.13399

12. Whiting DR, Guariguata L, Weil C, Shaw J. IDF Diabetes Atlas: Global Estimates of the Prevalence of Diabetes for 2011 and 2030. Diabetes Res Clin Pract (2011) 94(3):311-21. doi: 10.1016/j.diabres.2011.10.029

13. Boulton A. Strengthening the International Diabetes Federation (IDF). Diabetes Res Clin Pract (2020) 160:108029. doi: 10.1016/j.diabres.2020. 108029

14. Calgaroto NS, Thomé GR, da Costa P, Baldissareli J, Hussein FA, Schmatz R, et al. Effect of Vitamin D3 on Behavioural and Biochemical Parameters in Diabetes Type 1-Induced Rats. Cell Biochem Funct (2014) 32(6):502-10. doi: $10.1002 / \mathrm{cbf} .3044$

15. Neelankal John A, Iqbal Z, Colley S, Morahan G, Makishima M, Jiang FX. Vitamin D Receptor-Targeted Treatment to Prevent Pathological Dedifferentiation of Pancreatic $\beta$ Cells Under Hyperglycaemic Stress. Diabetes Metab (2018) 44(3):269-80. doi: 10.1016/j.diabet.2017.07.006

16. Uruska A, Zozulinska-Ziolkiewicz D, Niedzwiecki P, Pietrzak M, WieruszWysocka B. TG/HDL-C Ratio and Visceral Adiposity Index may be Useful in Assessment of Insulin Resistance in Adults With Type 1 Diabetes in Clinical Practice. J Clin Lipidol (2018) 12(3):734-40. doi: 10.1016/ j.jacl.2018.01.005

17. He J, He S, Liu K, Wang Y, Shi D, Chen X. The TG/HDL-C Ratio Might Be a Surrogate for Insulin Resistance in Chinese Nonobese Women. Int J Endocrinol (2014) 2014:105168. doi: 10.1155/2014/105168

18. Bothou C, Beuschlein F, Spyroglou A. Links Between Aldosterone Excess and Metabolic Complications: A Comprehensive Review. Diabetes Metab (2020) 46(1):1-7. doi: 10.1016/j.diabet.2019.02.003

19. Salgado AL, Carvalho L, Oliveira AC, Santos VN, Vieira JG, Parise ER. Insulin Resistance Index (HOMA-IR) in the Differentiation of Patients With Non- 
Alcoholic Fatty Liver Disease and Healthy Individuals. Arq Gastroenterol (2010) 47(2):165-9. doi: 10.1590/\$0004-28032010000200009

20. Perry RC, Baron AD. Impaired Glucose Tolerance. Why Is It Not a Disease? Diabetes Care (1999) 22(6):883-5. doi: 10.2337/diacare.22.6.883

21. American Diabetes Association. (2) Classification and Diagnosis of Diabetes. Diabetes Care (2015) 38 Suppl:S8-s16. doi: 10.2337/dc15-S005

22. Elliott WJ. Systemic Hypertension. Curr Probl Cardiol (2007) 32(4):201-59. doi: 10.1016/j.cpcardiol.2007.01.002

23. Flegal KM, Kruszon-Moran D, Carroll MD, Fryar CD, Ogden CL. Trends in Obesity Among Adults in the United States, 2005 to 2014. JAMA (2016) 315 (21):2284-91. doi: 10.1001/jama.2016.6458

24. Ricci C, Schutte AE, Schutte R, Smuts CM, Pieters M. Trends in Alcohol Consumption in Relation to Cause-Specific and All-Cause Mortality in the United States: A Report From the NHANES Linked to the US Mortality Registry. Am J Clin Nutr (2020) 111(3):580-9. doi: 10.1093/ajcn/nqaa008

25. Young KA, Maturu A, Lorenzo C, Langefeld CD, Wagenknecht LE, Chen YI, et al. The Triglyceride to High-Density Lipoprotein Cholesterol (TG/HDL-C) Ratio as a Predictor of Insulin Resistance, $\beta$-Cell Function, and Diabetes in Hispanics and African Americans. J Diabetes Complications (2019) 33(2):11822. doi: 10.1016/j.jdiacomp.2018.10.018

26. Wang YL, Koh WP, Talaei M, Yuan JM, Pan A. Association Between the Ratio of Triglyceride to High-Density Lipoprotein Cholesterol and Incident Type 2 Diabetes in Singapore Chinese Men and Women. J Diabetes (2017) 9(7):68998. doi: 10.1111/1753-0407.12477

27. Gong R, Liu Y, Luo G, Liu W, Jin Z, Xu Z, et al. Associations of TG/HDL Ratio With the Risk of Prediabetes and Diabetes in Chinese Adults: A Chinese Population Cohort Study Based on Open Data. Int J Endocrinol (2021) 2021:9949579. doi: 10.1155/2021/9949579

28. Haluzík M, Parízková J, Haluzík MM. Adiponectin and Its Role in the Obesity-Induced Insulin Resistance and Related Complications. Physiol Res (2004) 53(2):123-9. doi: 10.2307/3434063
29. Bełtowski J. Adiponectin and Resistin-New Hormones of White Adipose Tissue. Med Sci Monit (2003) 9(2):Ra55-61.

30. Morton RE. Cholesteryl Ester Transfer Protein and its Plasma Regulator: Lipid Transfer Inhibitor Protein. Curr Opin Lipidol (1999) 10(4):321-7. doi: 10.1097/00041433-199908000-00006

31. Wacker M, Holick MF. Sunlight and Vitamin D: A Global Perspective for Health. Dermatoendocrinol (2013) 5(1):51-108. doi: 10.4161/derm.24494

32. Nakano T, Cheng Y-F, Lai C-Y, Hsu L-W, Chang Y-C, Deng J-Y, et al. Impact of Artificial Sunlight Therapy on the Progress of Non-Alcoholic Fatty Liver Disease in Rats. J Hepatol (2011) 55(2):415-25. doi: 10.1016/j.jhep. 2010.11.028

33. Erem AS, Razzaque MS. Vitamin D-Independent Benefits of Safe Sunlight Exposure. J Steroid Biochem Mol Biol (2021) 213:105957. doi: 10.1016/ j.jsbmb.2021.105957

Conflict of Interest: The authors declare that the research was conducted in the absence of any commercial or financial relationships that could be construed as a potential conflict of interest.

Publisher's Note: All claims expressed in this article are solely those of the authors and do not necessarily represent those of their affiliated organizations, or those of the publisher, the editors and the reviewers. Any product that may be evaluated in this article, or claim that may be made by its manufacturer, is not guaranteed or endorsed by the publisher.

Copyright $\odot 2022 \mathrm{Liu}$, Gong, Luo, Li, Li, Yang and Wei. This is an open-access article distributed under the terms of the Creative Commons Attribution License (CC BY). The use, distribution or reproduction in other forums is permitted, provided the original author(s) and the copyright owner(s) are credited and that the original publication in this journal is cited, in accordance with accepted academic practice. No use, distribution or reproduction is permitted which does not comply with these terms. 